\title{
An experimental study on the $\mathrm{ZnO} /$ sapphire layered surface acoustic wave device
}

\author{
Tsung-Tsong Wu and Wei-Shan Wang \\ Institute of Applied Mechanics, National Taiwan University, Taipei, Taiwan
}

(Received 7 November 2003; accepted 27 June 2004)

\begin{abstract}
In this study, $\mathrm{ZnO}$ thin films with different thicknesses were deposited on C-plane sapphire substrates by radio-frequency magnetron sputtering. Properties of interdigital transducer/ $\mathrm{ZnO} /$ sapphire layered surface acoustic wave device including surface wave velocity, electromechanical coupling coefficient, and propagatiion loss were measured and discussed. Combining the effective permittivity approach and the coupling-of-modes (COM) model, the dispersion characteristics and frequency responses of the $\mathrm{ZnO} /$ sapphire layered structure were simulated. By substituting the measured parameters into the COM model, the simulated frequency response has shown good agreement with the experimental results. () 2004 American Institute of Physics. [DOI: 10.1063/1.1785842]
\end{abstract}

\section{INTRODUCTION}

In recent years, there has been an increasing interest in developing surface acoustic wave (SAW) devices operating frequencies. In order to obtain high-frequency SAW devices, a substrate material with hih acoustic velocity is desired. By depositing a piezoelectric film on top of a high acoustic velocity substrate, the purpose of high frequency can be achieved without fabricating submicron electrodes. The dispersion relations and electromechanical coupling of a layered SAW such as $\mathrm{ZnO} /$ sapphire or $\mathrm{ZnO}$ /diamond have been calculated, ${ }^{1-4}$ and the experimental results on layered SAW filters were reported. ${ }^{2,5-8} \mathrm{ZnO}$ thin film has been widely used for layered SAW devices due to its high coupling coefficient. On the other hand, sapphire $\left(\mathrm{Al}_{2} \mathrm{O}_{3}\right)$ substrate is an attractive material because of its high acoustic velocity and a relatively lower cost. Therefore, in this paper, we adopted the $\mathrm{ZnO}$ thin film on a sapphire substrate as a promising structure for high-frequency layered SAW devices.

In case of the layered structure, the phase velocity of SAW is dispersive; thus this effect must be taken into in designing SAW devices. Koike, Shimoe, and Ieki ${ }^{9}$ adopted Smith's equivalent circuit model to simulate the $\mathrm{ZnO} / \mathrm{R}$-sapphire SAW filter. Hachigo and Malocha ${ }^{10}$ calculated the null frequency bandwidth of $\mathrm{ZnO} /$ diamond/Si layered SAW device using the $\delta$ fucntion model. Emanetoglu et $a l^{11}$ presented an analysis of a $\mathrm{ZnO} / \mathrm{R}$-sapphire layered SAW device based on the Green's function. Wu and Chen ${ }^{12}$ employed the effective permittivity approach to simulate the frequency response of a $\mathrm{ZnO} /$ diamond/Si layered SAW device. Chen, $\mathrm{Wu}$, and $\mathrm{Chou}^{13}$ calculated the frequency response of IDT (interdigital transducer)/ZnO/R-sapphire SAW filter by combining the effective permitivity approach and the coupling-of-modes (COM) model. ${ }^{14}$

In this paper, details on fabricating IDT/ZnO/sapphire layered SAW structures are given. SAW properties, including dispersion of surface wave velocity, electromechanical coupling coefficient, and propagation loss, are measured. The measured dispersions of the SAW properties are compared with those simulated using the effective permiittivity approach. ${ }^{12}$ The measured frequency response of the layered SAW filter is compared with the simulated one by using the method proposed in Ref. 13.

\section{FABRICATION OF ZnO/SAPPHIRE SAW DEVICES}

There have been many techniques for growing $\mathrm{ZnO}$ thin film, such as sputtering, ${ }^{2,15}$ metal organic chemical vapor deposition (MOCVD), ${ }^{11}$ and molecular beam epitaxy (MBE). ${ }^{16}$ In this study, $\mathrm{ZnO}$ thin films were deposited on a sapphire (0001) substrate using the radio-frequency (rf) magnetron sputtering system. A $100 \mathrm{~mm}$ diameter $\times 3 \mathrm{~mm}$ thick $\mathrm{ZnO}$ disk with purity of $99.99 \%$ was used as the target. The distance between the substrate and the target was about $13 \mathrm{~cm}$. The sputtering chamber was evacuated to a pressure below $2 \times 10^{-4} \mathrm{~Pa}$ before sputtering. Prior to presputtering, the substrate was heated to $220^{\circ} \mathrm{C}$, and the ambient gases of mixed argon and oxygen were introduced into the chamber. In order to remove any contamination, the target was presputtered for nearly $20 \mathrm{~min}$ under $200 \mathrm{~W}$ rf power before the actual sputtering started. The thickness of the $\mathrm{ZnO}$ thin film is varied in proportion to the deposition time. The sputtering parameters are listed in Table I. Under the sputtering conditions summarized in Table I, C-axis oriented polycrystalline films could be grown on sapphire (0001) substrates. The surface roughness and thickness of the $\mathrm{ZnO}$ thin films were measured by a surface profiler. The crystalline structure and orientation of the $\mathrm{ZnO}$ films were confirmed by the $\mathrm{x}$-ray diffraction (XRD) measurement. Scanning electron microscopy (SEM) was employed to observe the surface morphology.

TABLE I. ZnO sputtering conditions.

\begin{tabular}{lc}
\hline \hline Target & \\
Target-substrate distance & $99.99 \% \mathrm{ZnO}$ \\
Sputtering gas & $\sim 13 \mathrm{~cm}$ \\
Substrate temperature & $\mathrm{Ar} \% \mathrm{O}_{2} 50 \%$ \\
RF power & $220^{\circ} \mathrm{C}$ \\
Deposition time & $200 \mathrm{~W}$ \\
\hline
\end{tabular}




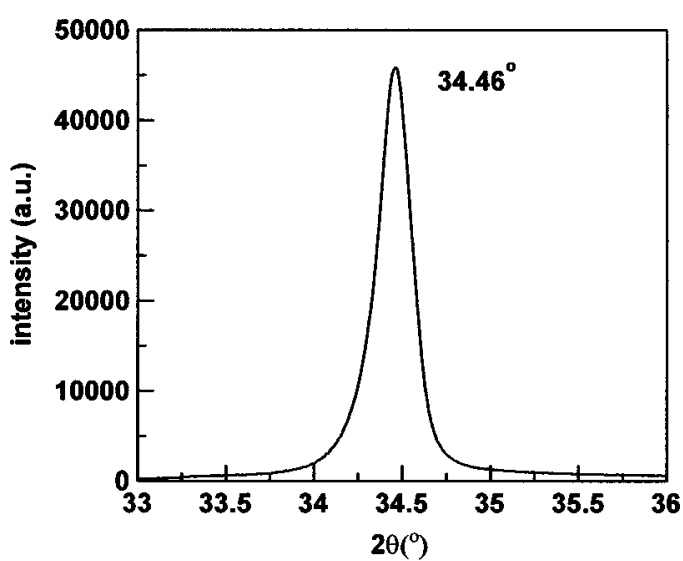

FIG. 1. An XRD pattern of the $\mathrm{ZnO}$ thin film.

Figure 1 shows the XRD diffraction pattern of the $\mathrm{ZnO}$ thin film on sapphire (0001) substrates. The angular peak position of the $\mathrm{ZnO}$ film is $2 \theta=34.46^{\circ}$. The pattern indicates that (0002) preferred orientation of the $\mathrm{ZnO}$ film could be obtained under the sputtering parameters we used. Figure 2 shows a SEM image of the cross section of $\mathrm{ZnO} / \mathrm{C}$-sapphire structure. As can be seen from the figure, the growth direction of $\mathrm{ZnO}$ is along the preferred $\mathrm{C}$-axis, and the thickness can also be estimated from the figure.

The layered SAW strcuture used in our study is IDT/ZnO/sapphire, as shown in Fig. 3. After the deposition of $\mathrm{ZnO}$ thin film, normal interdigital transducers were fabricated on the $\mathrm{ZnO}$ thin films. Aluminum was deposited using electron beam evaporation, followed by conventional lithography and lift-off process, to form the IDT fingers. The IDT design parameters are summarized in Table II.

\section{SAW PROPERTIES OF ZnO/SAPPHIRE LAYERED STRUCTURES}

The properties of the $\mathrm{ZnO} /$ sapphire SAW devices were characterized by using a probe station and a network analyzer (Agilent 8714ES). In our study, all measurements were under on-wafer conditions. The dispersion characteristics of the IDT/ZnO/C-plane sapphire layered structure are calculated based on the effective permittivity approach, as studied in Refs. 12 and 13. Material constants used for the calcula-

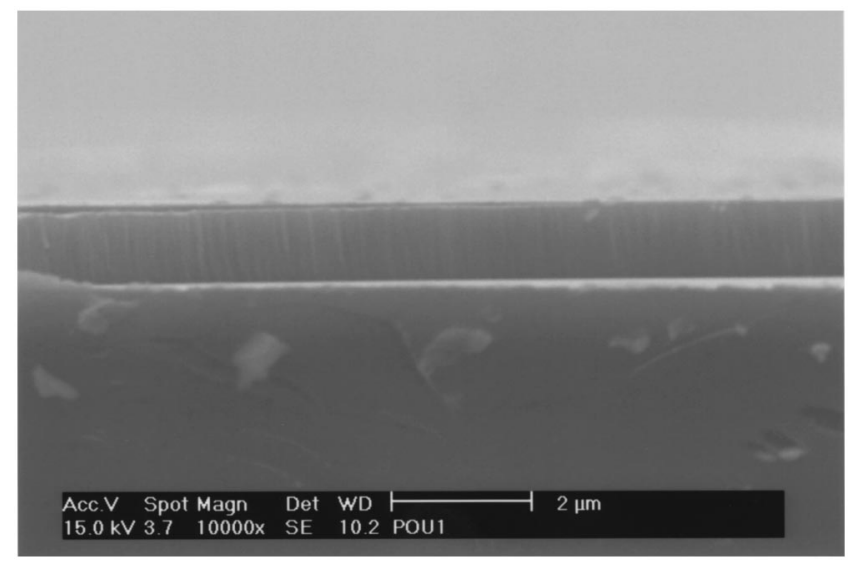

FIG. 2. A SEM picture of $\mathrm{ZnO}$ thin film on C-plane sapphire.

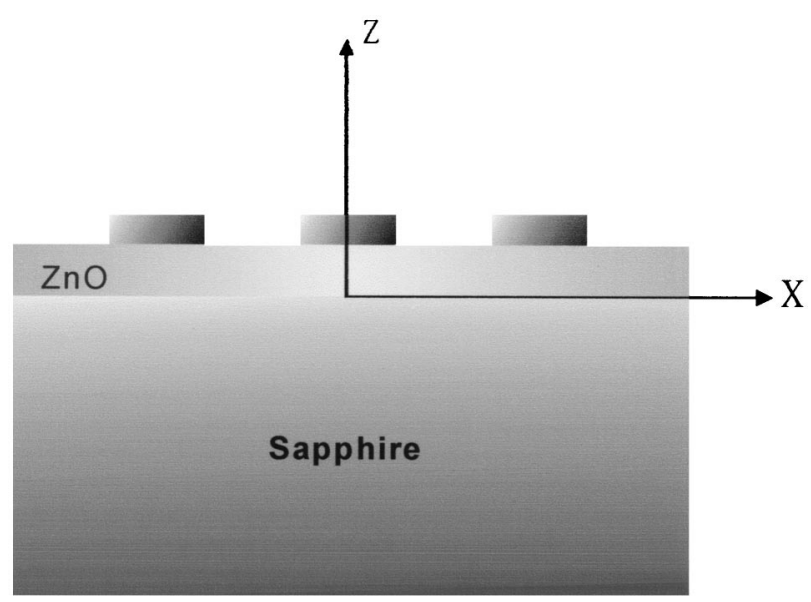

FIG. 3. Coordinates of a planner-layered half space.

tions were taken from those listed in Ref. 17. SAW properties such as surface wave velocity, electromechanical coupling coefficient, and propagation loss are reported and discussed in this section.

\section{A. Surface wave velocity}

In this study, $\mathrm{ZnO}$ films with various thicknesses (1-2 $\mu \mathrm{m})$ were sputtered on the sapphire substrates. IDT with wavelengths 8 and $12 \mu \mathrm{m}$ were designed and fabricated on $\mathrm{ZnO}$ films. These different designs can thus form the dispersion relation experimentally. Figure 4 shows the phase velocity dispersion of the product of the frequency and the thickness of the $\mathrm{ZnO}$ layer $\left(f h_{\mathrm{ZnO}}\right)$. In the figure, the solid line represents theoretical values of the first higher-order mode (first mode) of Rayleigh wave, and the dashed one stands for the second Rayleigh mode. Both of these higherorder modes are the so-called Sezawa modes. The circles and triangles in the figure are experimental values of the first and second Rayleigh modes, respectively. The phase velocity $V_{p}$ is obtained from the relation $V_{p}=f_{0} \lambda$, where $f_{0}$ is the center frequency and $\lambda$ is the periodicity of the IDT. In Fig. 4, the fundamental mode (zeroth) of the Rayleigh SAW is not shown, because of the electromechanical coupling coefficient of the zeroth Rayleigh mode almost approaches zero, as the thicker dashed line shown in Fig. 5. Due to such a low coupling coefficient, it is hard to excite this mode; thus it is very hard to measure. Some experimental values (circles and triangles in Fig. 4) are close to each other due to the small differences of the deposited $\mathrm{ZnO}$ films. The similar thickness was also the reason why we cannot form the complete dispersion curves experimentally. It is noted that measured phase velocities are slightly lower than the calculated ones,

TABLE II. IDT design parameters.

\begin{tabular}{lc}
\hline \hline Wavelength & $12 \mu \mathrm{m}$ \\
Finger space & $3 \mu \mathrm{m}$ \\
Aperture & $840 \mu \mathrm{m}$ \\
IDT pairs & 20,40 \\
\hline \hline
\end{tabular}




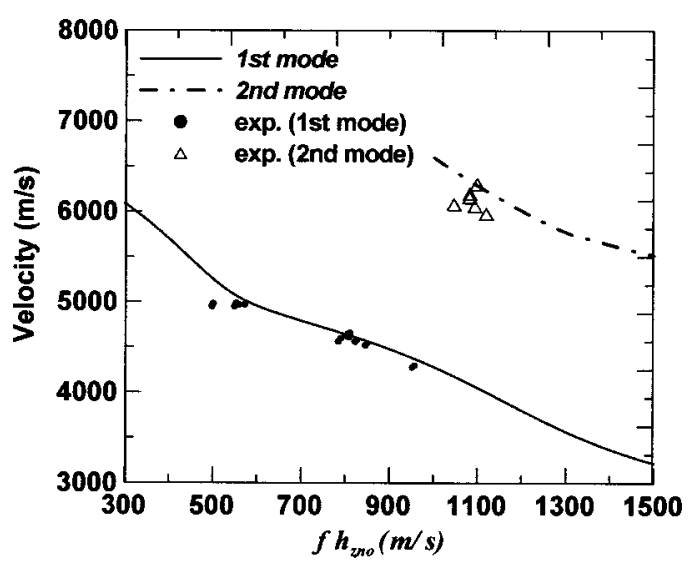

FIG. 4. Phase velocity dispersions of an IDT/ZnO/C-plane sapphire layered structure.

especially for second Rayleigh mode; however, the trend of the experimental results coincide well with the theoretical values.

\section{B. The electromechanical coupling coefficient}

Theoretical value of the electromechanical coupling coefficient is usually approximated as

$$
K_{s}^{2}=2 \frac{v_{0}-v_{m}}{v_{0}},
$$

where $v_{0}$ is the free surface velocity (open-circuited velocity) and $v_{m}$ is the surface wave velocity with metallized surface (short-circuited velocity). In this paper, $K_{s}^{2}$ was calculated by using the exact equation ${ }^{18}$

$$
K_{s}^{2}=2 \Gamma_{s} \varepsilon_{s}^{(\infty)},
$$

where $\varepsilon_{s}^{\infty}$ is the effective permittivity at infinite slowness and the function $\Gamma_{s}$ is defined in Ref. 19. The main difference of Eqs. (1) and (2) in layered structure was discussed in detail in Ref. 12.

Figure 5 shows the electromechanical coupling coefficient of IDT/ZnO/C-plane sapphire layered structure as a function of $f h_{\mathrm{ZnO}}$. The thicker dashed line represents theoretical values of the zeroth Rayleigh mode, the solid line is the

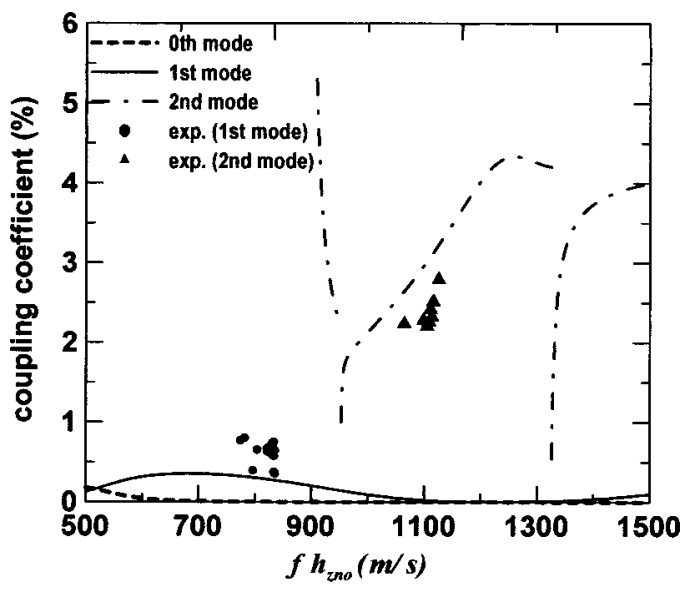

FIG. 5. Electromechanical coupling coefficient dispersions of an IDT/ZnO/C-plane sapphire layered structure.

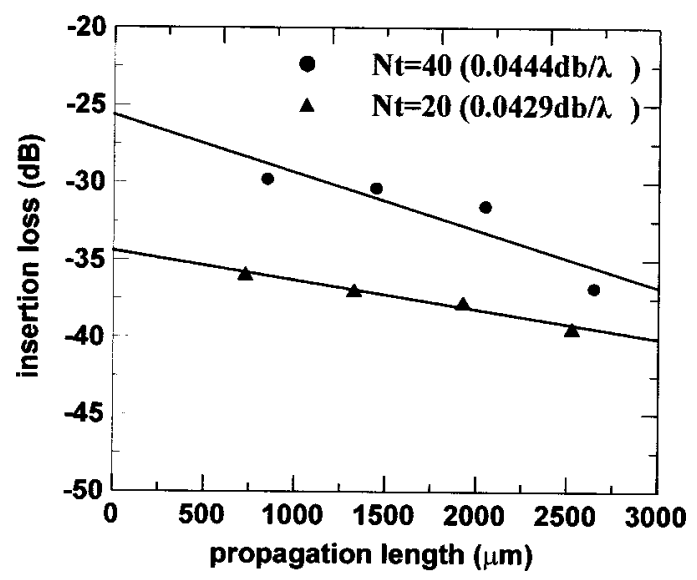

FIG. 6. Plot of insertion loss of SAW with various propagation distances.

first mode of Rayleigh wave, and the thinner dashed line is the second Rayleigh mode. The electomechanical coupling coefficient of the second Rayleigh mode can be as high as $4.3 \%$ at $f h_{\mathrm{ZnO}} \cong 1250 \mathrm{~m} / \mathrm{s}$, while the phase velocity is about $5885 \mathrm{~m} / \mathrm{s}$. This means IDT/ZnO/C-plane sapphire is a promising structure for SAW devices if we choose a proper value of $f h_{\mathrm{ZnO}} . K_{s}^{2}$ can be determined from the equation ${ }^{20}$

$$
K_{s}^{2}=\frac{\hat{G}_{a}}{8 f_{0} C_{T} N^{2}}
$$

where $\hat{G}_{a}$ is the radiation conductance, $f_{0}$ is the center frequency, $C_{T}$ is the total electrode capacitance of the IDT, and $N$ is the number of IDT finger pairs. By measuring the S11 (reflection) parameter and using Eq. (3), the experimental value of $K_{s}^{2}$ was calculated. The circles and triangles in Fig. 5 represent experimental values of the first and second Rayleigh modes, respectively. As mentioned before, it is hard to excite the zeroth Rayleigh mode experimentally; thus we cannot measure the electromechanical coupling coefficient of this mode. It is noted the deposited $\mathrm{ZnO}$ films were about 1-2 $\mu \mathrm{m}$; thus the circles and triangles in Fig. 5 form small range of dispersion curves and are close to each other. Once we deposit $\mathrm{ZnO}$ films with larger range than we have done in this paper, the dispersion curves described by experimental values in Figs. 4 and 5 will be more complete.

\section{Propagation loss $\gamma$}

Propagation loss attenuates the surface acoustic wave amplitude and affects the insertion loss of SAW devices, in particular, at high frequencies. For conventional half-space SAW devices, the crystal is well polished and the loss due to surface imperfections is not significant. For layered structures, the surface with deposited $\mathrm{ZnO}$ film is not so perfect that the propagation loss must be considered for practical SAW devices. The propagation loss was estimated from insertion losses at center frequencies with different propagation distances. ${ }^{2,21}$ Figure 6 shows the propagation losses of the first Rayleigh mode as a function of the propagation distances. The design parameters of the IDT listed in Table II were used to construct different propagation lengths which were the distances between the centers of the input and out- 
put IDTs. For 40 pairs of IDT, four kinds of propagation distances range between 846 and $2646 \mu \mathrm{m}$, and a $600 \mu \mathrm{m}$ interval was designed. For 20 pairs of IDT, the minimum and maximum propagation distances were 726 and $2526 \mu \mathrm{m}$, respectively, and the interval was also $600 \mu \mathrm{m}$. The $\mathrm{ZnO}$ thin film was $1.206 \mu \mathrm{m}$ thick and the center frequencies was $413 \mathrm{MHz}$. When the slopes of Fig. 6 were determined, the propagation losses of $0.0444 \mathrm{~dB} / \lambda$ for 40 pairs of IDT and $0.0429 \mathrm{~dB} / \lambda$ for 20 pairs of IDT were obtained. The results show that the propagation losses are almost the same irrespective of different IDT pairs. This indicates that once the sputtering parameters of a design is set, the uniform $\mathrm{ZnO}$ film could be deposited and the propagation loss of the whole SAW device could be treated as a constant, for example, $\gamma=0.04 \mathrm{~dB} / \lambda$.

\section{FREQUENCY RESPONSES OF ZnO/SAPPHIRE LAYERED STRUCTURES}

The COM model has been widely used in modeling nondispersive SAW devices for many years. Based on the formulations, the effects of propagation loss, electrode reflections, electrical transduction, acoustic reception, thin film loss, and the distributed finger capacitance can be included. Unlike half-space SAW devices, some of the COM parameters utilized for analyzing a layered SAW become frequency dependent due to the phase velocity dispersion, and have to be modified accordingly. Frequency dependences of the COM parameters such as the reflection parameter, the surface wave velocity, and the transduction parameter can be calculated by employing the effective permittivity approach. Details of calculating the COM parameters for a dispersive layered SAW were studied by $\mathrm{Chen}, \mathrm{Wu}$, and Chou. ${ }^{13} \mathrm{In}$ this paper, we adopted the method as in Ref. 13, that is, combining the effective permittivity approach and the COM model, to simulate the frequency response of the two-port IDT/ZnO/C-plane sapphire layered SAW device.

In general, some calculated parameters used in the simulation model, such as coupling coefficients and surface velocities, are not totally matched with those measured from fabricated SAW devices. Furthermore, there is no formula to estimate propagation loss for a layered SAW device. Therefore, measurements of these key parameters are needed. By substituting these measured parameters into the COM model, the simulation results can be more precise. In this section, frequency responses of the numerical and experimental results are compared and discussed. To compare the stimulation result with the measured frequency response, the time gating technique was employed to remove the effect of the electromagnetic feedthrough.

In this study, the period of the IDT is $12 \mu \mathrm{m}$, the number of the IDT pairs is 40 and the thickness of the IDT elctrodes is $714 \AA$. The measured thickness of the $\mathrm{ZnO}$ film is $h_{\mathrm{ZnO}}$ $=1.206 \mu \mathrm{m}$ and the center frequency of the first Rayleigh mode is $413.25 \mathrm{MHz}$. The measured surface wave velocity is $4959 \mathrm{~m} / \mathrm{s}$ and the simulated center frequency is $404 \mathrm{MHz}$. Figure 7 shows the frequency responses. The thick line represents the experimental result, and the thinner one is simulated frequency response. From the figure, one finds that the

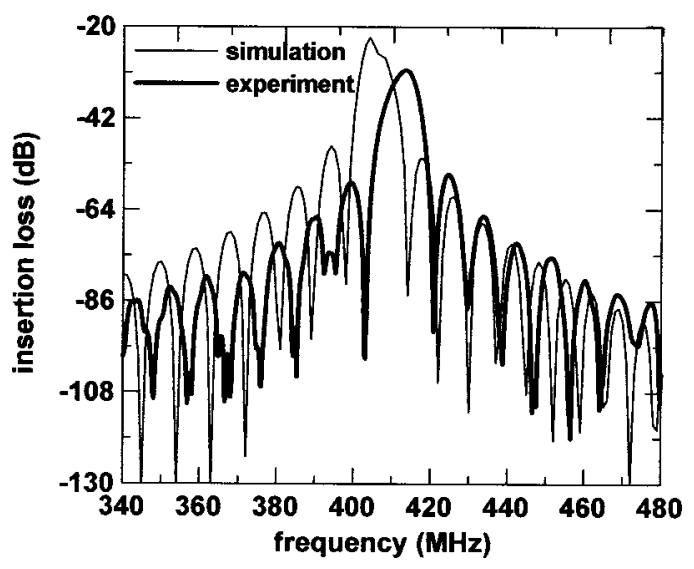

FIG. 7. Measured S21 characteristic (thicker line) and the calculated frequency response based on the COM model (thinner line).

insertion loss of the calculated one is about $7 \mathrm{~dB}$ smaller than that of the measured one. In addition, the center frequency of the simulated one is about $10 \mathrm{MHz}$ lower than the measured one. Possible reasons for the discrepancy between the measured center frequency and the simulated one are as follows.

(1) One is the difference in the material constants for $\mathrm{ZnO}$ between single crystals used for calculations and the deposited thin film.

(2) Another is the use of (0001) $\mathrm{ZnO} / /(0001)$ sapphire to analyze the layered SAW device. We note that the sputtered $\mathrm{ZnO}$ thin film was not actually $\mathrm{C}$-axis oriented, though very close to it, as shown in Fig. 1. This is a possible reason which causes the variations of velocity and then the frequency responses between the numerical and experimental results.

(3) In the COM model, the perturbed surface wave velocity $v_{R}$ due to short circuit gratings ${ }^{22,23}$ was considered while analyzing the layered SAW devices. This is an approximated formula and the properties of the electrode material also may affect the parameter $v_{R}$. The discrepancy between the measured and the simulated velocities may be due to the overprediction of the perturbed surface wave velocity $v_{R}$. Figure 8 shows a comparison between

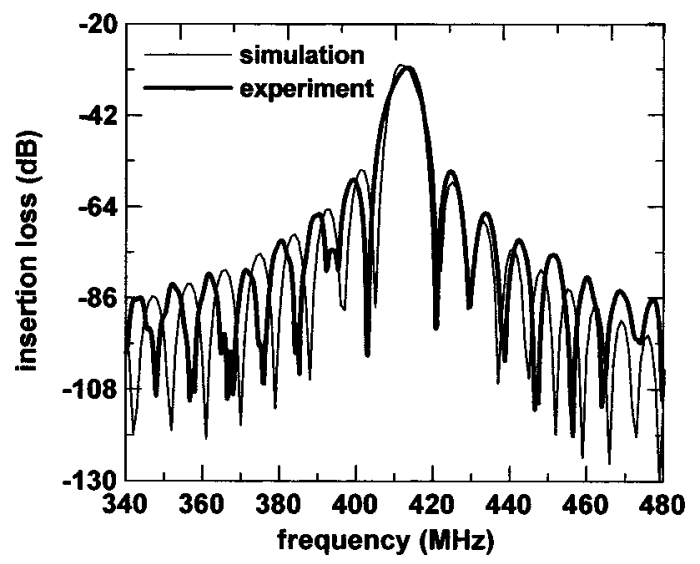

FIG. 8. Measured S21 characteristic (thicker line) and the calculated frequency response based on the COM model modified with measured parameters (thinner line). 
the calculated and measured frequency responses in which the measured propagation loss and phase velocity are adopted in the numerical simulation. One finds that by substituting the measured surface velocity into the COM model, the tuned value of the simulated center frequency becomes $414 \mathrm{MHz}$ which is very close to the measured one of $413 \mathrm{MHz}$. By incorporating the measured propagation loss into the simulated model, the insertion loss of the filter is very close to that of measured one $-30.35 \mathrm{~dB}$.

\section{CONCLUSION}

Good $\mathrm{C}$-axis oriented $\mathrm{ZnO}$ thin films were deposited on C-plane sapphire substrates by rf- sputtering IDT/ZnO/sapphire layered SAW structures were successfully fabricated in this study. Then, SAW properties, including surface wave velocity, electromechanical coupling coefficient, and propagation loss, were measured. The dispersion characteristics were calculated by using the effective permittivity approach. The measured data of the phase velocity and the electromechanical coupling coefficient were compared and discussed with the calculated dispersion curves. The frequency response of the IDT/ZnO/sapphire layered structure was analyzed by combining the COM mdel and the effective permittivity approach. By substituting the measured parameters into the COM model, the simulated and the modified frequency responses are in good agreement with the measured ones.

\section{ACKNOWLEDGMENT}

The authors acknowledge the financial support to this research from the National Science Council of Taiwan through Grant No. NSC90-2212-E-002-156.
${ }^{1}$ G. S. Kino and R. S. Wagers, J. Appl. Phys. 44, 1480 (1973).

${ }^{2}$ T. Mitsuyu, S. Ono, and K. Wasa, J. Appl. Phys. 51, 2464 (1980).

${ }^{3}$ E. L. Adler and L. Solie, IEEE Ultrason. Symp. 341 (1995).

${ }^{4}$ H. Nakahata, A. Hachigo, K. Higaki, S. Fujii, S. Shikata, and N. Fujimori, IEEE Trans. Ultrason. Ferroelectr. Freq. Control 42, 362 (1995).

${ }^{5}$ H. Nakahata, K. Higaki, S. Fujii, S. A. Hachigo, H. Kitabayashi, K. Tanabe, Y. Seki, and S. Shikata, IEEE Ultrason. Symp. 361 (1995).

${ }^{6}$ M. S. Wu, A. Azuma, T. Shiosaki, and A. Kawabata, J. Appl. Phys. 62, 2482 (1987).

${ }^{7}$ M. Kadota and M. Minakata, Jpn. J. Appl. Phys., Part 1 37, 2923 (1998).

${ }^{8}$ C. R. Gorla, N. W. Emanetoglu, S. Liang, Y. Lu, M. Wraback, and H. Shen, J. Appl. Phys. 85, 2295 (1999).

${ }^{9}$ J. Koike, K. Shimoe, and H. Ieki, Jpn. J. Appl. Phys., Part 1 32, 2337 (1993).

${ }^{10}$ A. Hachigo and D. C. Malocha, IEEE Trans. Ultrason. Ferroelectr. Freq. Control 45, 660 (1998).

${ }^{11}$ N. W. Emanetoglu, G. Patounakis, S. Liang, C. R. Gorla, R. Wittstruck, and Y. Lu, IEEE Trans. Ultrason. Ferroelectr. Freq. Control 48, 1389 (2001).

${ }^{12}$ T. T. Wu and Y. Y. Chen, IEEE Trans. Ultrason. Ferroelectr. Freq. Control 49, 142 (2002).

${ }^{13}$ Y. Y. Chen, T. T. Wu, and T. T. Chou, J. Phys. D 37, 120 (2004).

${ }^{14}$ B. P. Abbott, Ph. D. thesis, Department of Electrical Engineering, University of Central Florida Orlando, Florida, USA (1989).

${ }^{15}$ H. Ieki and M. Kadota, IEEE Ultrason. Symp. 281 (1999).

${ }^{16}$ Y. F. Chen, D. M. Bagnall, H. J. Koh, K. T. Park, K. Hiraga, Z. Zhu, and T. Yao, J. Appl. Phys. 84, 3912 (1998).

${ }^{17}$ B. A. Auld, Acoustic Fields and Waves in Solids (Malabar, Florida, 1990).

${ }^{18}$ H. Matthews, Surface Wave Filters: Design, Construction, and Use (Wiley, New York, 1977), p. 66, Eq. (2.30).

${ }^{19}$ D. P. Morgan, Surface Wave Devices for Signal Processing (Elsevier, New York, 1991).

${ }^{20}$ W. R. Smith, H. M. Gerard, J. H. Collins, T. M. Reeder, and H. J. Shaw, IEEE Trans. Microwave Theory Tech. 17, 856 (1969).

${ }^{21}$ S. Shikata, H. Nakahata, K. Higaki, A. Hachigo, N. Fujimori, Y. Yamamoto, N. Sakairi, and Y. Takashi, IEEE, Ultrason. Symp. 277 (1993).

${ }^{22}$ T. Thorvaldsson, IEEE Ultrasonics Symp. 91 (1989).

${ }^{23}$ B. P. Abbott, IEEE Ultrasonics Symp. 5 (1991). 